\title{
Orienting molecules via an ir and uv pulse pair: Implications for coherent Raman spectroscopy
}

\author{
Alexei V. Sokolov, ${ }^{1}$ Kevin K. Lehmann, ${ }^{2}$ Marlan O. Scully, ${ }^{1,3}$ and Dudley Herschbach ${ }^{1,4}$ \\ ${ }^{1}$ Texas A\&M University, College Station, Texas 77843, USA \\ ${ }^{2}$ University of Virginia, Charlottesville, Virginia 22904, USA \\ ${ }^{3}$ Princeton University, Princeton, New Jersey 08544, USA \\ ${ }^{4}$ Harvard University, Cambridge, Massachusetts 02138, USA
}

(Received 15 January 2009; published 4 May 2009)

\begin{abstract}
Spatial orientation of molecules is a pervasive issue in chemical physics and, by breaking inversion symmetry, has major consequences in nonlinear optics. In this paper, we propose and analyze an approach to molecular orientation. This extracts from an ensemble of aligned diatomic molecules (equally $A B$ and $B A$, relative to the $\mathcal{E}$ vector) a subensemble that is oriented (mostly $A B$ or $B A$ ). Subjecting an aligned molecule to a tailored infrared (ir) laser pulse creates a pair of coherent wave packets that correlate vibrational phase with the $A B$ or $B A$ orientation. Subsequent, suitably phased ultraviolet (uv) or visible pulses dissociate one of these vibrational wave packets, thereby "weeding out" either $A B$ or $B A$ but leaving intact the other orientation. Molecular orientation has significant implications for coherent Raman spectroscopy. In the absence of orientation, coherence between vibrational levels is generated by a pair of laser pulses off which a probe pulse is scattered to produce a signal. Orientation allows direct one-photon ir excitation to achieve (in principle) maximal Raman coherence.
\end{abstract}

DOI: 10.1103/PhysRevA.79.053805

PACS number(s): 42.50.Hz, 33.80.-b

\section{INTRODUCTION}

Elucidation of molecular structure and spectra, experimental and theoretical, has built up over many decades a vast body of stereochemical [1] and optical [2] data and lore. More recently, molecular beam and laser developments have provided direct access to dynamical aspects of molecular orientation in gas phase single collisions [3].

Since untamed molecules are not only randomly oriented but rotate freely, obtaining the experimental capability to align or orient a molecular axis has required means to restrict molecular rotation [4]. Pioneering experiments [5,6] exploited the fact that symmetric top molecules (having a threefold or higher axis, as in $\mathrm{CH}_{3} \mathrm{I}$ ), in rotational states having finite angular momentum along the symmetry axis, precess rather than tumble. State selection by a focusing electric field thus suffices to pick out molecules with substantial intrinsic orientation of the symmetry axis. Orientation of polar molecules other than symmetric tops by an electric field was long considered impractical, because rotational tumbling averages out the dipole moment in first order and hence greatly weakens interaction with an electric field [7]. However, generating beams via supersonic expansion drastically lowers rotational energy, enabling molecular tumbling to be quenched without use of inordinately high field strengths. The molecular axis is then confined to a restricted angular range, as it swings to and from about the field direction $[8,9]$. The pendular states thereby created are coherent linear superpositions of the field-free rotational states and can be made highly directional.

Intense pulsed laser fields have provided a kindred variety of pendular states, aligned rather than oriented. This utilizes the induced dipole moment created by nonresonant interaction of a laser with the anisotropic polarizability of the molecule $[10,11]$, producing a double-well potential as indicated in Fig. 1. The pendular alignment occurs adiabatically and persists only during the duration of the laser pulse, if it is long compared with the librational and rotational periods. However, field-free alignment can be obtained by use of an ultrashort pulse that produces recurrent alignment due to revivals of coherent rotational wave packets $[12,13]$. Pendular alignment can be turned into orientation in several ways that break the double-well symmetry. If the laser-induced barrier between the $A B$ and $B A$ orientations is high enough to make the two lowest pendular states nearly degenerate in energy, imposing a weak static field will split the degeneracy and produce a strong first-order Stark effect [14]. Another approach, not yet implemented, is to employ an off-resonant laser pulse accompanied by its second harmonic to induce localization in the double well and thereby transform the adiabatic alignment of molecules into orientation during the pulse [15].

In this paper, we consider molecules that are initially aligned, but not oriented, and propose a means to attain orientation by exploiting selection of vibrational phase. This is now feasible by virtue of recent advances in laser technology, including the comb of phase coherent femtosecond pulses covering the spectrum from the ir to the uv. In Sec. II, we describe how ir pulses can be used to create a coherent vibrational wave packet. At very specific time(s) following this ir preparation, we use a uv or visible pulse to photodissociate molecules oriented the "wrong" way; see Figs. 2 and 3. This leaves behind an oriented ensemble of intact molecules. We illustrate for the $\mathrm{CO}$ molecule the effectiveness of this orientation selective excitation and its sensitivity to an optimal choice of the uv or visible pulse length. Further aspects of the "one-two punch" process are examined in the Appendix.

In Sec. III we discuss implications of molecular orientation for coherent Raman spectroscopies such as CARS and CSRS (anti-Stokes and Stokes). The key aspect pertains to the Raman coherence, specified by the density matrix element, $\rho_{b c}$. Typically, in the absence of orientation, this is 

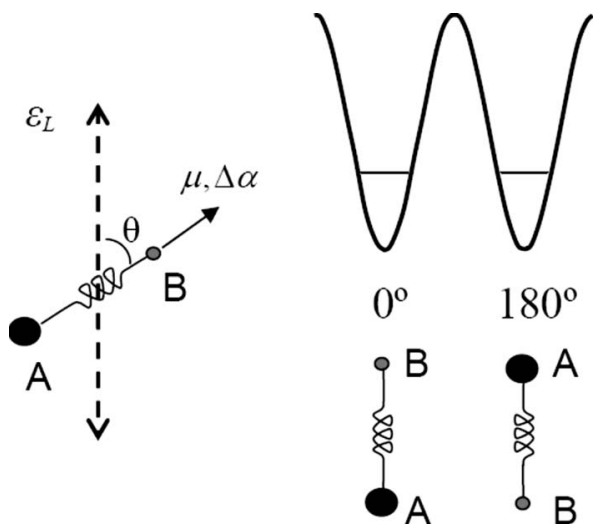

FIG. 1. Left: schematic depicting interaction of a polar diatomic molecule with a strong electric field, $\mathcal{E}_{L}$, produced by nonresonant laser light. The molecular axis is at an angle $\theta$ with respect to the field; $\mu$ denotes the permanent dipole moment, $\Delta \alpha=\alpha_{\|}-\alpha_{\perp}$ the anisotropy of the molecular polarizability. The rapid reversal of $\mathcal{E}_{L}$ averages out the permanent dipole interaction, proportional to $\mu \mathcal{E}_{L} \cos \theta$, but not the induced dipole interaction, proportional to $\Delta \alpha \mathcal{E}_{L}^{2} \cos ^{2} \theta$. Right: double-well potential produced by the induced dipole interaction. This potential restrains molecules in the lowest angular librational levels within the wells to pendular oscillations about the field direction. Because the polarizability does not distinguish between the two ends of the molecule, the wells are equivalent, so the $A B$ and $B A$ orientations occur equally.

generated by a pair of driving laser fields. Orientation allows use of direct one-photon ir excitation to attain (in principle) the maximal coherence, $\rho_{b c}=\frac{1}{2}$.

\section{ORIENTING MOLECULES VIA A COMBINATION OF INFRARED AND VISIBLE ULTRASHORT HIGH-POWER LASER PULSES}

\section{A. ir followed by uv or visible laser pulses}

Figure 3 illustrates the basic processes involved in using pulsed lasers to extract from an initial ensemble of aligned molecules a subensemble of oriented molecules. Use of strong resonant ir pulses creates coherent superpositions, $\Phi_{ \pm}$, of the ground and first excited vibrational states. These superposition states correspond to extending or compressing the bond length, depending on the orientation of the molecule relative to the ir driving field. Figure 3 is drawn for the case that the ir field points rightward and presumes that the charge distribution in the polar molecule is such that the $B$ end is subjected to a force in the field direction and the $A$ end opposite to the field direction. When the ir field reverses, the $A B$ orientation would be compressed and $B A$ extended. The essential feature is that the extended and compressed states, reflecting the vibrational phase, are correlated with the molecular orientation with respect to the phase of the ir field. The ability to manipulate the phase of the laser field relative to the molecular vibrational phase therefore gives us a grip on the molecular orientation (provided that the applied laser pulses are short compared to the tunneling time in the potential shown in Fig. 1). We note that, strictly speaking, aligned molecules can correspond to either coherent or incoherent

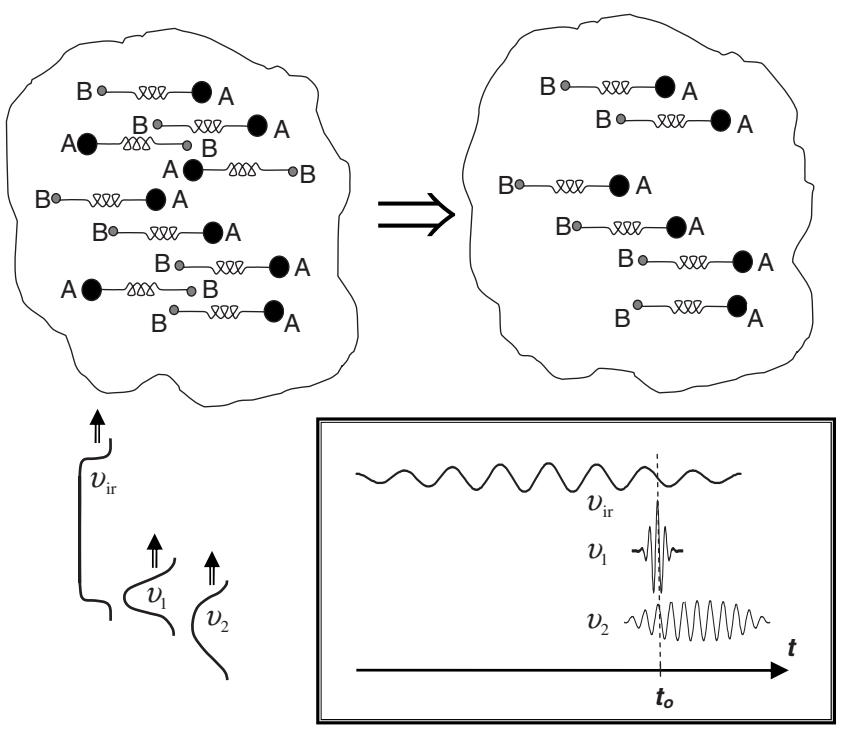

FIG. 2. Left: Ensemble of aligned molecules, equally populated with $A B$ and $B A$ orientations, is subjected to an ir laser pulse, followed by a few cycle uv or visible pulse $\nu_{1}$ and second laser pulse $\nu_{2}$ to selectively dissociate or ionize molecules of one orientation. Right: This leaves an ensemble of oriented molecules. The three pulses are shown schematically at the bottom left, where the ir pulse is top-hat and is directed such that its polarization is parallel to the molecular axis. The inset at the bottom right shows the three incident fields and their timing in more detail.

superpositions of oriented and antioriented molecular states, or to any degree of partial coherence. However, an initial coherence between the oriented and antioriented states plays no role in our following derivations.

Here we first outline an oversimplified analysis focusing on the wave function for the $A B$ diatom given by

$$
\Psi(X,\{\mathbf{r}\}, t)=\sum_{\alpha=g, 1,2} C_{\alpha}(t) \psi_{\alpha}(\{\mathbf{r}\}) \Phi_{\alpha}(X, t),
$$

where $X$ is the displacement of the internuclear separation from equilibrium, i.e., $X=R-R_{0}$ and $\{\mathbf{r}\}=\left\{\mathbf{r}_{1}, \mathbf{r}_{2}, \ldots\right\}$ denotes the vector position of the molecular electrons. The electronic and nuclear wave functions are given by $\psi_{\alpha}$ and $\Phi_{\alpha}$, for the ground and excited states as in Fig. 3. The electronic eigenstates are, of course, time independent, but it is convenient to describe the nuclear configuration in terms of timedependent basis states.

The ground-state nuclear wave function $\Phi_{g}(X, t)$ is of central interest. For the present proposes it suffices to focus on a superposition of the first two vibrational levels, denoted $|0\rangle$ and $|1\rangle$ in Fig. 3,

$$
\Phi_{g}(X, t)=a_{0}(t) \varphi_{0}(X)+a_{1}(t) \varphi_{1}(X) .
$$

The laser fields interact with the molecule via its permanent dipole moment, $\mu(R)$ and its polarizability components, $\alpha_{\|}(R)$ and $\alpha_{\perp}(R)$, respectively, parallel and perpendicular to the internuclear axis. As these are functions of the bond length, the dipole moment and polarizability vary somewhat as the molecule vibrates. The interaction energy is given by 


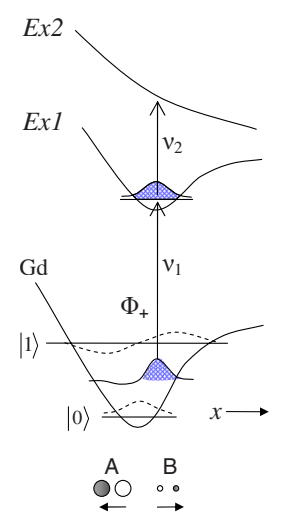

(a) Extended Molecule

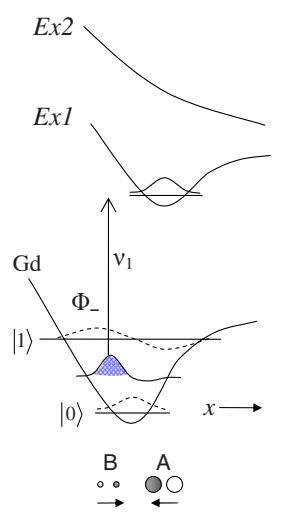

(b) Compressed Molecule

FIG. 3. (Color online) A strong ir pulse creates coherent superpositions, $\Phi_{ \pm}=2^{-1 / 2}(|0\rangle \pm|1\rangle)$, of the ground and first excited vibrational states. The $A B$ molecule is stretched (left) or compressed (right) depending on the orientation of the molecule relative to the ir driving field. After the ir pulse is switched off an ultrashort (2-3 fs) a uv or visible laser pulse is introduced that excites the $A B$ molecules (but not the $B A$ molecules) due to the Franck-Condon overlap integrals. The $A B$ are thus excited from the ground electronic state manifold Gd to the excited state manifold Ex1 and subsequently to a higher state Ex2 that leads to dissociation or ionization of $A B$ (but no $B A$ ) molecules.

$$
-\mu \mathcal{E}_{L}(t) \cos \theta-\frac{1}{2} \mathcal{E}_{L}^{2}(t)\left(\alpha_{\|} \cos ^{2} \theta+\alpha_{\perp} \sin ^{2} \theta\right),
$$

where the laser field $\mathcal{E}_{L}(t)=\mathcal{E}_{0} \cos \left(\nu_{L} t+\phi\right)$, with $\nu_{L}$ the laser frequency, $\phi$ the phase. Considering for now only the permanent dipole term, we take account of the time dependence by

$$
\mu(t)=\mu_{0}+(\partial \mu / \partial R)_{0} X(t)+\cdots .
$$

Here the derivative represents an effective charge, $e_{\text {eff }}$ $=\mu_{1}=(\partial \mu / \partial R)_{0}$, and the zero subscripts indicate quantities evaluated at the equilibrium bond distance, where $X=0$. The probability amplitudes $a(t)$ in Eq. (1) may be calculated via the interaction Hamiltonian, wherein the potential term is

$$
V(t)=-e_{\mathrm{eff}} X(t) \mathcal{E}_{L}(t) \cos \theta .
$$

The $\mu_{0}$ term of Eq. (4) is omitted because averaged out by the rapid reversal of $\mathcal{E}_{L}(t)$. For simplicity, we consider the ir pulse to have a "top-hat" profile, switched on abruptly at $t$ $=0$ and off at $t=t_{0}$. At resonance where the ir laser frequency equals the molecular vibrational fundamental frequency, $\omega_{0}$, we find

$$
\dot{a}_{0}=-\frac{1}{2} \Omega a_{1}, \quad \dot{a}_{1}=\frac{1}{2} \Omega a_{0},
$$

where we set $\phi=\frac{\pi}{2}$ and

$$
\Omega=\bar{\Omega} \cos \theta=e_{\mathrm{eff}}\langle 1|X| 0\rangle \mathcal{E}_{0} \cos \theta / \hbar
$$

is the Rabi frequency. At the switch-off time $t_{0}$, we have
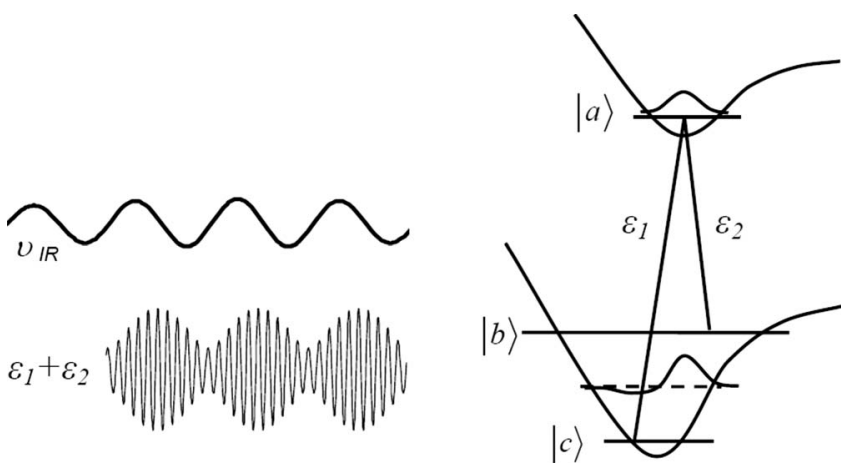

FIG. 4. Orientation-dependent excitation of ir-driven molecular vibration by a pair of quasisinusoidal fields resonant with an excited electronic state. The difference frequency of the pair of fields matches the ir field but can be phase shifted with respect to it. Note that, to be consistent with customary notation in quantum optics [17], the ground-state vibrational levels denoted by $|0\rangle$ and $|1\rangle$ in Fig. 3 are denoted by $|c\rangle$ and $|b\rangle$ here and in subsequent figures.

$$
a_{0}\left(t_{0}\right)=\cos \left(\Omega t_{0} / 2\right), a_{1}\left(t_{0}\right)=\sin \left(\Omega t_{0} / 2\right),
$$

where for perfect alignment $\cos \theta= \pm 1$, so $\Omega= \pm \bar{\Omega}$. Hence, by adjusting the pulse duration so that $\bar{\Omega} t_{0}=\pi / 2$, we obtain

$$
\Phi_{g}\left(X, t_{0}\right)=\Phi_{ \pm}\left(X, t_{0}\right)=\frac{1}{\sqrt{2}}\left[\varphi_{0}(X) \pm \varphi_{1}(X)\right],
$$

the oppositely phased, coherent superpositions indicated in Fig. 3 with $\Phi_{+}$pertaining to the $0^{\circ}$ orientation and $\Phi_{-}$to $180^{\circ}$.

In similar fashion, as shown in the Appendix, the probability amplitudes associated with Eq. (1) for the transition from the ground to the excited electronic state $(\mathrm{Gd} \rightarrow \mathrm{Ex} 1$ in Fig. 3) may be obtained from

$$
\begin{aligned}
& \dot{C}_{g}^{( \pm)}=-i \Omega_{ \pm} C_{1}^{( \pm)}, \\
& \dot{C}_{1}^{( \pm)}=-i \Omega_{ \pm} C_{g}^{( \pm)},
\end{aligned}
$$

where the \pm designations again pertain to the $0^{\circ}$ and $180^{\circ}$ orientations. The Rabi frequencies for the electronic transition, $\Omega_{+}$, are proportional to the Franck-Condon factors, so if these impose $\Omega_{+}>\Omega_{-}$, when the uv pulse arrives (see Fig. 2 , inset), there will be preferential dissociation of $A B$ molecules $\left(0^{\circ}\right.$ orientation). Repeating the process at a rate that equals the ir frequency, $\omega_{0}$, will thus deplete the $A B$ orientation but not the $B A$ orientation.

Although convenient for our initial heuristic description, presuming an abrupt "top-hat" style switching on and off of laser pulses is clumsy and unrealistic. However, replacing these pulse shapes with more realistic ones (Gaussian or other smooth shapes) is not expected to make any qualitative difference. As pointed out above, what is more important (and rather difficult to attain in practice) is the timing of the visible or uv pulses with respect to the ir phase. Figure 4 exhibits a much preferable method. This employs, in addition to the ir laser, a pair of visible or uv laser fields $\mathcal{E}_{1}$ and $\mathcal{E}_{2}$. These are resonant with an electronically excited state $|a\rangle$ 
and have their beat frequency, $\nu_{1}-\nu_{2}=\omega_{0}$, equal to the ground-state ir frequency but shifted in phase (with respect to the ir field) by $90^{\circ}$. The beat frequency field thus combines with the ir field in driving the molecular vibration. In practice, the ir field can be obtained from the two visible or uv laser fields from a difference frequency generation; then the relative phase is automatically fixed and can be adjusted easily. Thereby one or the other of the $0^{\circ}$ or $180^{\circ}$ molecular orientations can be promoted to the excited state $|a\rangle$, then subjected to another pulsed laser field that produces photodissociation or ionization.

In the frequency domain representation, this method (of Fig. 4) is analogous to electromagnetically induced transparency (EIT) and to the dark- and bright-state physics of the $\Lambda$ system $[16,17]$. As in Fig. 3, molecules with the desired orientation are prepared via ir in a vibrational superposition state and are therefore immune to electronic excitation, whereas the other orientation is not. In this scheme, the real transition amplitudes induced by fields $\mathcal{E}_{1}$ and $\mathcal{E}_{2}$ interfere with opposite signs for \pm orientations. By judicious selection of the relative amplitudes of $\mathcal{E}_{1}$ and $\mathcal{E}_{2}$, one can, in principle, completely cancel the excitation for one of the orientations. Thus we will only excite to state 1 and subsequently ionize or dissociate molecules of the other orientation.

There is an important question that Fig. 4 helps to answer. It pertains to the optimal pulse duration for the uv laser $\left(v_{1}\right.$ in the scenario of Figs. 2 and 3$)$ that will produce maximal orientation. If the pulse duration is much longer than the molecular vibrational period, it cannot produce appreciable orientation-selective excitation, and will simply selectively deplete one of the ground states. If the pulse is very short, however, the laser spectrum becomes correspondingly broad, so does not allow selective excitation into only one vibrational level $|a\rangle$ of the excited electronic state. Other vibration levels will then become involved, so most any superposition of the ground-state vibrational wave functions will reach the excited state within the excitation bandwidth, again precluding orientation-selective excitation. The optimal pulse duration is expected to coincide with the duration of beats in Fig. 4 , which is roughly equal to half of the molecular vibrational period (or ir period). In Sec. II C, this is indeed the result we find from a calculation for the $\mathrm{CO}$ molecule, modeled using harmonic vibrational potentials for the ground and excited electronic states.

The convenient presumption of perfect prealignment is likewise unrealistic but more problematic. Any alignment method will produce a substantial range of orientations about $0^{\circ}$ and $180^{\circ}$. The range corresponds to the librational amplitude of the pendular states associated with the double-well potential of Fig. 1. The ratio $\Delta \alpha E^{2} / B$, with $B$ the molecular rotational constant (inversely proportional to the moment of inertia), governs the librational amplitudes as well as the height of the barrier separating the wells and the number and location of the pendular states bound within the wells. References $[9,10]$ provide nomograms from which these quantities and the relative populations of the pendular states in a Boltzmann gas ensemble can be obtained. Typically, unless the gas temperature is exceptionally low, several pendular states will be populated. However, usually the librational motions are much slower than the bond stretching vibrations.

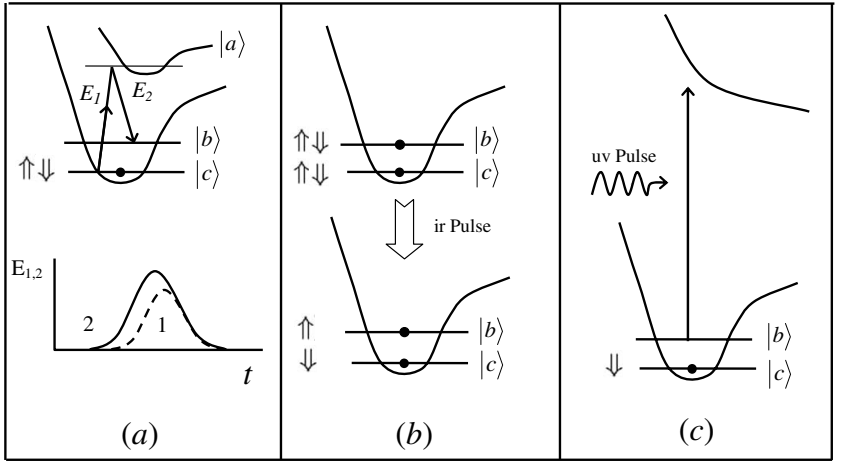

FIG. 5. (a) Aligned molecules are prepared in a coherent superposition of vibrational states via fractional STIRAP pulse arrangement involving pulses 1 and 2. (b) Next the molecules are driven by a $\frac{\pi}{2}$ ir pulse which leaves oriented molecules in the first vibrational state and antioriented molecules are left in vibrational ground state. (c) Finally the molecules in the $|b\rangle$ are photoassociated by a uv laser pulse and the remaining subensemble is oriented as indicated.

Thus, we can approximate the orientation angle $\theta$ as frozen. In the scenario considered in Figs. 2-4, the Rabi frequencies will differ somewhat for each $\theta$; a specified laser pulse will then have a corresponding spread in pulse area (e.g., a nominal $\pi / 2$ ir pulse will have an area of $\frac{\pi}{2} \cos \theta$ for each $\theta$ ). The range in orientations about $0^{\circ}$ and $180^{\circ}$ hence will reduce the $A B$ vs $B A$ contrast ratio below that for perfect prealignment. As a quantitative estimate and examination of ameliorating possibilities would require elaborate analysis, for our present purpose we simply note this situation.

\section{B. Fractional STIRAP followed by ir laser pulses}

Figure 5 presents another expeditious method to convert alignment into orientation. This employs a variant of the stimulated Raman with adiabatic passage (STIRAP) technique [18]. Instead of using the visible or uv fields to promote the molecules into excited electronic states, as in Fig. 3, we can use them to produce a Raman excitation, followed, preceded by, or concurrent with the ir pulse. Ordinary STIRAP uses a "counterintuitive" sequence of two Ramanresonant visible or uv laser pulses, applying pulse 2 before 1 in order to transfer population from $|b\rangle$ to $|c\rangle$. In fractional STIRAP, we also turn on pulse 2 first but turn off both pulses at the same time.

As indicated in Fig. 5(a), molecules initially in the ground vibrational state $|c\rangle$, equally likely in either the $0^{\circ}$ (upward arrow) or $180^{\circ}$ (downward arrow) orientation, are subjected to a STIRAP pulse pair. This creates a coherent superposition, so converts the initial state vector,

$$
\left|\Phi_{g}\left(0^{-}\right)\right\rangle=|c\rangle \otimes \frac{1}{\sqrt{2}}[|\Uparrow\rangle+|\Downarrow\rangle],
$$

into

$$
\left|\Phi_{g}\left(0^{+}\right)\right\rangle=\frac{1}{\sqrt{2}}[|b\rangle+|c\rangle] \otimes \frac{1}{\sqrt{2}}[|\Uparrow\rangle+|\Downarrow\rangle] .
$$


Thereby the populations of $|c\rangle$ and $|b\rangle$, the ground and first excited vibrational states, become equal although both still have a 50\%-50\% mixture of the two orientations. Please note that here there is no sign difference between the Raman excitation amplitudes for up and down orientations (as opposed to the case of one-photon excitation). This is because the transition amplitude is due to even order (polarizability) tensor. Next, as indicated in Fig. 5(b), a $\frac{\pi}{2}$ ir pulse with duration $\tau$ is applied that induces a correlation between vibrational excitation and orientation, leaving molecules in $|b\rangle$ in the $0^{\circ}$ orientation and those in $|c\rangle$ in the $180^{\circ}$ orientation,

$$
\left.\left|\Phi_{g}(\tau)\right\rangle_{\pi / 2}=\frac{1}{\sqrt{2}}[|c\rangle|| \Uparrow\rangle+|b\rangle|\Downarrow\rangle\right] .
$$

Finally, as shown in Fig. 5(c), molecules in the $|b\rangle$ state are photodissociated or ionized by resonant excitation, leaving intact the $|c\rangle$ state in the $180^{\circ}$ orientation.

More generally, we can detune the pair of applied visible or uv fields off the one-photon resonance, while still keeping them Raman resonant. Then this excitation can be followed (or preceded) by an ir pulse, phased such that it would add to the vibrational excitation for one particular molecular orientation and subtract from the other.

Since the $\pi / 2$ pulse has the key role, we outline the derivation of Eq. (12). The pulse drives the coherent superposition attained in Eq. (11b) via

$$
\left|\Phi_{g}(\tau)\right\rangle=e^{-i / \hbar H_{\mathrm{ir}}}\left|\Phi_{g}(0)\right\rangle,
$$

where

$$
H_{\text {ir }}=\frac{\hbar \Omega}{2}\left[e^{-i \phi}|b\rangle\left\langle c\left|+e^{i \phi}\right| c\right\rangle\langle b|\right],
$$

with $\Omega=\bar{\Omega} \cos \theta$ the Rabi frequency defined in Eq. (7) and $\phi$ the ir phase, as yet unspecified. The eigenstates of Eq. (14) corresponding to $\cos \theta= \pm 1$ are given by

$$
| \pm\rangle_{\phi}=\frac{1}{\sqrt{2}}\left[|b\rangle \pm e^{i \phi}|c\rangle\right] .
$$

After expressing $|b\rangle$ and $|c\rangle$ in terms of $| \pm\rangle_{\phi}$, applying Eq. (13) to Eq. (11b) with $\Omega= \pm \bar{\Omega}$, and setting $\phi=\pi / 2$, we obtain

$$
\begin{aligned}
\left|\Phi_{g}(\tau)\right\rangle= & \frac{1}{\sqrt{2}}\left[\frac{1}{\sqrt{2}}\left(\cos \frac{\bar{\Omega} \tau}{2}-\sin \frac{\bar{\Omega} \tau}{2}\right)|b\rangle\right. \\
& \left.+\frac{1}{\sqrt{2}}\left(\cos \frac{\bar{\Omega} \tau}{2}+\sin \frac{\bar{\Omega} \tau}{2}\right)|c\rangle\right]|\Uparrow\rangle \\
& +\frac{1}{\sqrt{2}}\left[\frac{1}{\sqrt{2}}\left(\cos \frac{\bar{\Omega} \tau}{2}+\sin \frac{\bar{\Omega} \tau}{2}\right)|b\rangle\right. \\
& \left.+\frac{1}{\sqrt{2}}\left(\cos \frac{\bar{\Omega} \tau}{2}-\sin \frac{\bar{\Omega} \tau}{2}\right)|c\rangle\right]|\Downarrow\rangle .
\end{aligned}
$$

Choosing the pulse area $\bar{\Omega} \tau=\pi / 2$ then yields Eq. (12).
Please note that, for clarity, in Eqs. (11), (12), and (16) we have only shown the positive superposition of $0^{\circ}$ and $180^{\circ}$ orientations. In the scenario described above, the negative superposition would behave exactly the same way. If a strong aligning ac field is turned on adiabatically, the $J=M=0$ ground state will evolve into a symmetric superposition of the ground oriented and antioriented states, while the $J=1$, $M=0$ state will evolve to the antisymmetric combination. Thus, the coherence between the oriented and antioriented states will be proportional to the difference in initial population of these two free rotor states. Any more detailed consideration of the issues relating to the molecules' initial rotational state, and the coupling between two opposite orientations in an aligned molecular ensemble (due to tunneling), is beyond the scope of our discussion here. As noted in Sec. II A, none of our results depend on whether we start with a coherent or incoherent superposition of oriented and antioriented molecular states.

\section{Example: Orientation of the CO molecule}

A key issue for attaining orientation by the method we propose is the duration of the electronic excitation pulse, as noted in considering Fig. 4. We have assumed that it can be made short compared with the vibrational period, so that the excitation pulse always finds the bond either extended or contracted. However, we have also assumed that the excitation pulse has sufficiently narrow bandwidth that it can selectively excite those molecules with an extended bond length. These are conflicting requirements. The usual FranckCondon approximation ignores the dependence on the vibrational bond length of the electronic transition moment between ground and excited electronic states. In that limit, the excitation rate of a delta function excitation pulse will be totally unselective of orientation, because the electronic excitation probability will be independent of the ground state vibrational wave function. To achieve selective excitation, we must have an electronic excitation pulse that is at least comparable in duration to the excited state vibrational period. For such a pulse, we cannot ignore the vibrational motion in the ground state during the pulse. A pulse much longer than the vibrational period would average over several cycles and thus pump both orientations of the molecule approximately equally.

To examine what might be achieved with a pulse of intermediate duration, we have estimated the orientationdependent selectivity for uv excitation of the $\mathrm{CO}$ molecule from the ground electronic state, $X^{1} \Sigma^{+}$, to the first excited electronic state, $A^{1} \Pi$. This requires evaluating the FranckCondon factors $\left(\mathcal{F}_{\mathrm{FC}}\right)$ between the ir induced coherent superpositions, $\Phi_{g}=\Phi_{ \pm}$, of the lowest two vibrational levels of the $X$ electronic state and vibrational levels of the $A$ state. We use harmonic oscillator functions: the vibrational wave numbers are $\omega=2170$ and $\omega^{\prime}=1516 \mathrm{~cm}^{-1}$ and equilibrium bond distances $R_{e}=1.1281$ and $R_{e}^{\prime}=1.2341 \AA$, respectively, for the $X$ and $A$ states [19]; the $0-0$ excitation wave number is $T_{00}$ $=64746 \mathrm{~cm}^{-1}$. The Franck-Condon factors, $\mathcal{F}_{\mathrm{FC}}(X, v$ $\left.\rightarrow A, V^{\prime}\right)=\left(f_{v v^{\prime}}\right)^{2}$, with $f_{v v^{\prime}}$ the overlap integral of the normalized $X$ and $A$ harmonic oscillator wave functions, give 
the relative intensity of vibrational bands. Closure requires, for all levels that contribute appreciably, $\Sigma_{v^{\prime}} \mathcal{F}_{\mathrm{FC}}\left(v^{\prime}, v\right)$ $=\Sigma_{v} \mathcal{F}_{\mathrm{FC}}\left(v, v^{\prime \prime}\right)=1$. We consider the molecule initially in the $X$ state vibrational superposition of Eq. (9), $\Phi_{ \pm}$ $=a_{0} \varphi_{0} \pm a_{1} \varphi_{1} \exp (2 \pi i c \omega t)$, subjected to a pulse with electric field amplitude $\mathcal{E}(t)$ that excites vibrational levels of the $A$ state with the amplitudes $b_{v^{\prime}} \exp \left[2 \pi i c\left(T_{00}+v^{\prime} \omega^{\prime}\right) t\right]$. If the pulse is weak enough, the excitation can be calculated by first-order perturbation theory which gives

$$
b_{v^{\prime}}=\frac{\mu_{X A}}{i \hbar}\left[a_{0} f_{0 v^{\prime}} \hat{F}\left(\Delta E_{0 v^{\prime}}\right) \pm a_{1} f_{1 v^{\prime}} \hat{F}\left(\Delta E_{1 v^{\prime}}\right)\right] \text {. }
$$

Here $\mu_{X A}$ is the electronic transition dipole matrix element and $\hat{F}\left(\Delta E_{v v^{\prime}}\right)$ is the Fourier transform of the laser field amplitude evaluated at the transition frequency $\Delta E_{v v^{\prime}}$ $=2 \pi c\left(T_{00}+v^{\prime} \omega^{\prime}-v \omega\right) / \hbar$. The total number of molecules transferred to the $A$ state will be proportional to $\Sigma_{v^{\prime}}\left|b_{v^{\prime}}\right|^{2}$.

From this calculation, we see that differential excitation of the extended and compressed coherent vibrational states, $\Phi \pm$, arises from interference of the two terms appearing in Eq. (17). The effect depends strongly on the Franck-Condon amplitudes $f_{v v^{\prime}}$ and the spectrum, shape, and duration of the excitation pulse. If we consider simply transitions to the $v^{\prime}$ $=0$ level of the $A$ state from the $v=0$ and $v=1$ levels of the $X$ state, we find the $\mathcal{F}_{\mathrm{FC}}$ factors are

$$
\left(f_{00^{\prime}}\right)^{2}=0.123, \quad\left(f_{10^{\prime}}\right)^{2}=0.211 .
$$

In contrast, for transitions to the $v^{\prime}=0$ level of the $A$ state from the coherent vibrational superpositions $\Phi_{+}$and $\Phi_{-}$of the $X$ state, the $\mathcal{F}_{\mathrm{FC}}$ factors are given by

$$
\frac{1}{2}\left(f_{00^{\prime}}+f_{10^{\prime}}\right)^{2}=0.328, \quad \frac{1}{2}\left(f_{00^{\prime}}-f_{10^{\prime}}\right)^{2}=0.00528 \text {. }
$$

For a sufficiently short pulse, these factors would give the relative excitation probabilities. Thus, for the $\Phi_{+}$coherent state, corresponding to an stretched bond, constructive interference of the $\mathcal{F}_{\mathrm{FC}}$ amplitudes produces a modestly higher excitation probability than that for either the $v=0$ or 1 states, while for the $\Phi_{-}$coherence, corresponding to a compressed bond, destructive interference markedly reduces the excitation probability.

However, for excitation to other vibrational levels of the $A$ state, the results differ strikingly. For example, for the $v^{\prime}$ $=3$ level the relative probabilities for excitation from the $\Phi_{+}$ and $\Phi_{-}$states are 0.00045 and 0.343 , respectively: these are the reverse of the result for the $v^{\prime}=0$ level.

We have carried out calculations assuming either Gaussian or "top-hat" excitation pulse, with full width at half maximum $\Delta t$ and also with either the $0-0$ transition frequency or a frequency adjusted to optimize the orientation selectivity. Figure 6 shows the total population of the $A$ state produced by a Gaussian pulse, as a function of $\Delta t$ (specified in units of the $X$ state vibrational period). Dashed curves pertain to use of $T_{00}$ as the carrier wave number, as in Eq. (17); full curves use instead a value, $T_{\text {opt }}$, chosen to optimize the orientation selectivity. As expected, for a sufficiently

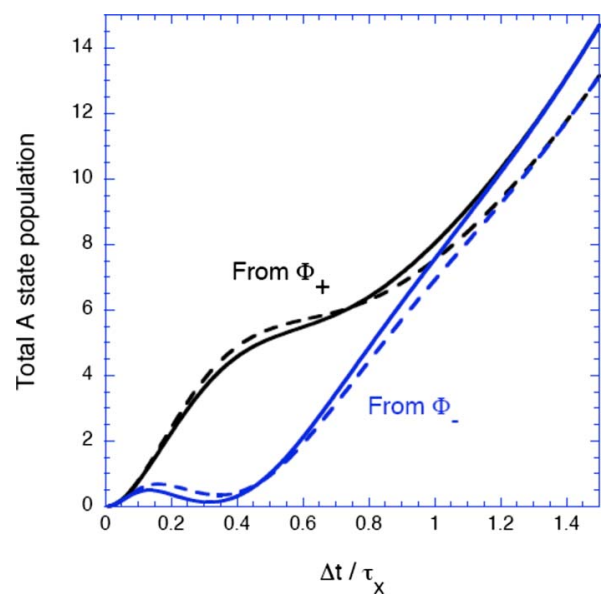

FIG. 6. (Color online) Total relative population, $\Sigma_{v^{\prime}}\left|b_{v^{\prime}}\right|^{2}$, of the excited $A$ state of $\mathrm{CO}$ resulting from excitation from the extended and compressed coherent vibrational superpositions, $\Phi_{+}$, of the lowest two vibrational levels of the ground $X$ state by a Gaussian pulse, as calculated from Eq. (17), plotted versus the ratio, $\Delta t / \tau_{X}$, of the FWHM of the pulse to the $X$ state harmonic vibrational period, $\tau_{X}=(c \omega)^{-1}=15.36 \mathrm{fs}$. Dashed curves pertain to using as the carrier wave number the value, $T_{00}$, for the $0-0$ transition; full curves instead use a value chosen to optimize the $P_{+} / P_{-}$ratio of Fig. 7 . To convert the ordinate scale to absolute units, the populations should be multiplied by $\left(\mu_{X A} \mathcal{E}_{0} / \hbar\right)^{2}$, where $\mathcal{E}_{0}$ is the electric field amplitude at the peak of the pulse. Reference [20] gives the transition dipole moment at $R_{e}$, from the $X$ state to $A$ states, as $\mu_{X A}$ $=0.899$ a.u. $=7.62 \times 10^{-30} \mathrm{Cm}$.

short excitation pulse (below 0.1 ), or for sufficiently long pulses (above 1.2), the total transition probability is the same for excitation of either the compressed $\left(\Phi_{-}\right)$or extended $\left(\Phi_{+}\right)$bond. Near $\Delta t=0.35$, however, the excitation probability from the compressed state has a pronounced minimum. As seen in Fig. 7, this produces a strong maximum in the ration $P_{+} / P_{-}$of the $A$ state population excited from the ex-

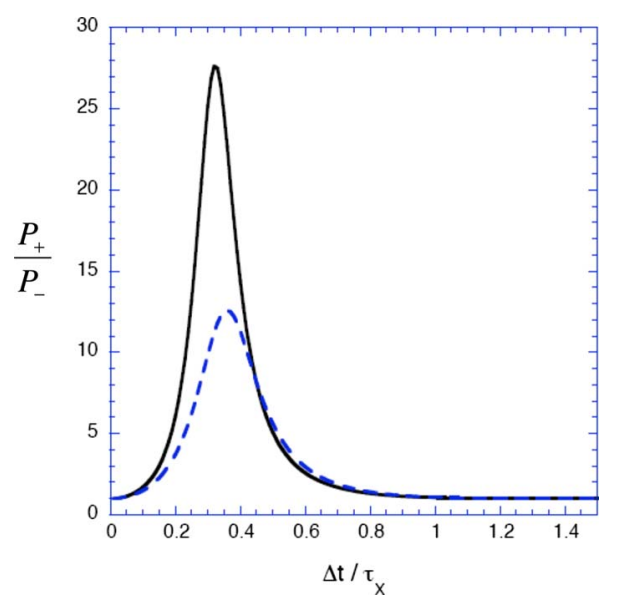

FIG. 7. (Color online) Ratio of populations plotted in Fig. 6, excited to the $A$ state of $\mathrm{CO}$ from the extended $\left(\Phi_{+}\right)$and compressed $\left(\Phi_{-}\right)$coherent vibrational superpositions of the ground $X$ state by a Gaussian pulse. Dashed and full curves correspond, as in Fig. 6 , to using as the carrier frequency either the $0-0$ transition frequency or an optimized value. 
tended and compressed coherent superpositions, $\Phi_{ \pm}$, of the $X$ state $v=0$ and 1 vibrations. Near $\Delta t=0.35$, excitation from the extended $\Phi_{+}$state is favored by a factor of about 13 ; with optimization of both the pulse width and carrier frequency, this factor grows to about 28, for $\Delta t=0.32$ and $T_{\text {opt }}=T_{00}$ $-0.3 \omega$. For this optimized Gaussian pulse, excitation from the $\varphi_{+}$state populates the $v^{\prime}=0$ and $v^{\prime}=1$ levels of the $A$ state about equally, whereas that appearing in any higher $v^{\prime}$ levels is more than 50-fold lower. In calculations using a "top-hat" (i.e., square wave) excitation pulse, we find qualitatively similar results, but the maximum $P_{+} / P_{-}$ratio reaches about 43 for an optimized pulse with $\Delta t=0.59$ and $T_{\text {opt }}=T_{00}-0.22 \omega$.

In summary, if diatomic molecules are strongly aligned by means of an intense Stark effect, an ir laser can create entanglement between the $A B$ and $B A$ orientations and the phase of the coherent vibrational superpositions of the $v=0$ and $v=1$ states. Our calculations for CO illustrate that a uv or visible pulse properly timed with respect to the vibrational coherence can indeed transform that entanglement into one between orientations and electronic excitation. With high fidelity, just one or the other of the orientations can be promoted to an electronically excited state, from which it can be efficiently removed by photodissociation or photoionization.

\section{COHERENT RAMAN SPECTROSCOPY VIA MOLECULAR ORIENTATION AND ir EXCITATION}

Coherent Raman scattering provides a useful tool for molecular identification. It substantially exceeds its incoherent (spontaneous) counterpart in the number of photons that can be generated from a given number of molecules. In particular, the ratio of coherent to incoherent Raman scattering goes as the square of the ground state density matrix element, $\rho_{b c}$ [21]. In an experiment where the prepared coherence is about $1 \%$ of its maximal value (which is rather high), the ratio of the number of scattered photons, coherent vs spontaneous, can still be as high as $10^{5}$ [22]. Preparation of a large molecular coherence is therefore an issue of crucial importance.

In this section we first briefly review two methods used, without the aid of molecular alignment or orientation, to produce coherence between vibrational levels and thereby strongly enhance sensitivity of Raman spectroscopy. Then we proceed to describe how molecular orientation can allow direct one-photon excitation of a large ground state coherence as quantified by the density matrix element $\rho_{b c}$.

\section{A. Conventional Raman spectroscopy review}

The usual way to generate the $\rho_{b c}$ coherence, illustrated in Fig. 8, uses a pair of laser pulses (1 and 2) differing in frequency by the vibrational frequency, $\omega_{b c}$. The parameter that determines the magnitude of the prepared Raman coherence is given by $\Omega_{R}=\Omega_{1} \Omega_{2} / \Delta$, where $\Omega_{1}=\mu_{a c} \mathcal{E}_{1} / \hbar$ and $\Omega_{2}$ $=\mu_{a c} \mathcal{E}_{2} / \hbar$ are the Rabi frequencies of the fields 1 and 2 (pump and Stokes), proportional to the dipole matrix elements $\mu_{\alpha \beta}$ between states $\alpha$ and $\beta$ and $\Delta$ is the detuning from the electronic resonance. When more than one excited state can be accessed through dipole interaction (as typically

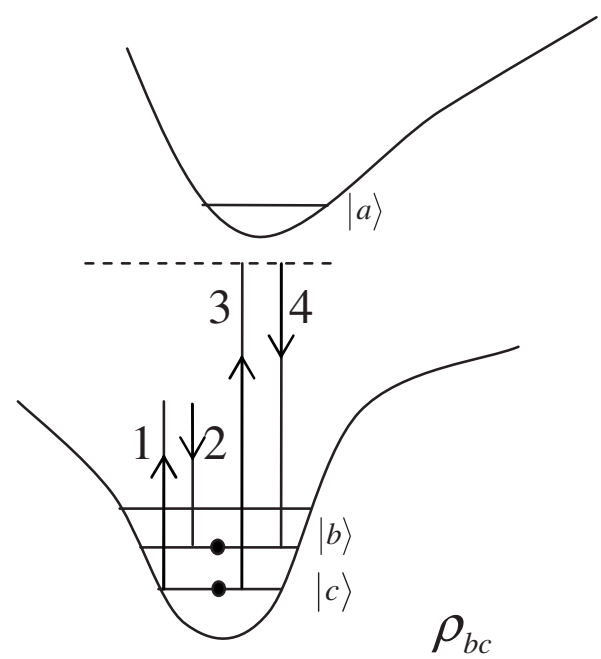

FIG. 8. Vibrational states $|b\rangle$ and $|c\rangle$ are prepared in a coherent superposition by fields $(1,2)$. The coherence is described by density matrix element $\rho_{b c}$. Coherent Raman radiation (4) is generated by scattering probe laser (3) off the oscillating molecule.

is the case), one needs to sum over the contributions of all such allowed transitions. Moreover, when $\Delta$ is large, other (counter-rotating) terms need to be included when calculating $\Omega_{R}$; these are all explicitly shown in Refs. [23,24]. The parameter $\Omega_{R}$ is often referred to as the effective Raman Rabi frequency. In the perturbative limit, and assuming Raman resonance, the density matrix element $\rho_{b c} \approx-\frac{i}{2} \Omega_{R} \tau_{1,2}$, where $\tau_{1,2}$ is the duration of pump and Stokes pulses ( 1 and 2), and therefore

$$
\rho_{b c} \approx-\frac{i}{2} \tau_{1,2} \Omega_{1} \Omega_{2} / \Delta .
$$

As shown in Ref. [21], the CARS scattering efficiency can be directly calculated from the value of $\rho_{b c}$. For a sample of $N$ molecules contained in a volume $V$, the ratio of signal-toprobe photon numbers

$$
\frac{n_{4}}{n_{3}}=\left[\frac{\pi}{2} \chi^{2} \frac{N \Delta z}{V}\right]^{2}\left|\rho_{b c}\right|^{2},
$$

where $\Delta z$ is the sample length, and $\chi$ is the light wavelength divided by $2 \pi$.

We next investigate the extent to which an infrared pulse, tuned to the molecular frequency $\omega_{\alpha \beta}$, can be used to generate the molecular coherence $\rho_{b c}$, via two photon ir processes, as shown in Fig. 9 below.

\section{B. Raman coherence via two photon ir excitation}

The other, less usual, means to generate Raman coherence employs direct two-photon ir excitation, illustrated in Fig. 9. We focus on simple diatomic molecules. The polarization dot product between the ir field and the molecule polarization is important. In particular, the interaction with the ir field may be written as 


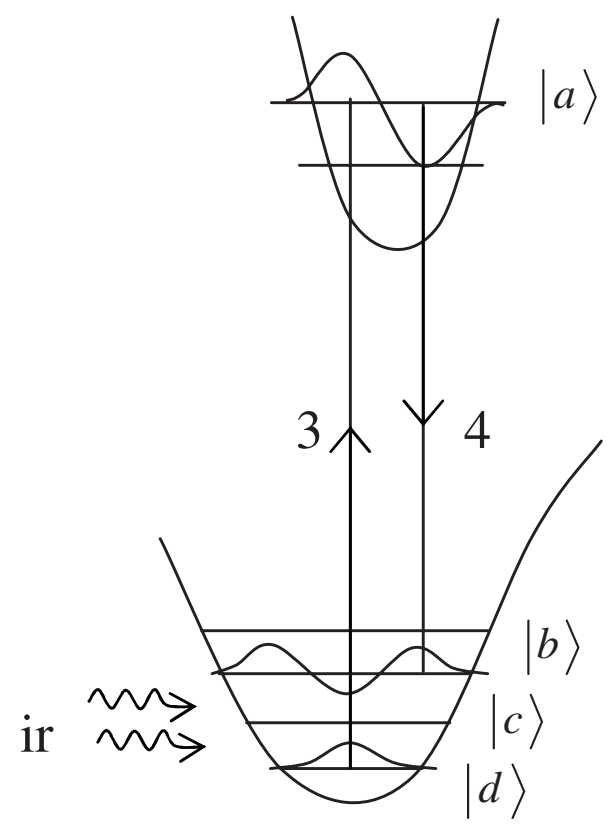

FIG. 9. Two infrared (ir) photons produce coherence between the $|d\rangle$ and $|b\rangle$ oscillator states. Probe laser (3) scatters off the $\rho_{b d}$ coherence generating coherent Raman signal (4). The oscillator wave functions of the ground state and (displaced) excited state are sketched to emphasize the role of Frank-Condon factors in resonant Raman scattering.

$$
V_{j}(t)=\hbar \Omega_{j}[|c\rangle\langle d|+| b\rangle\langle c|]_{j} e^{i \omega t+i \phi} e^{-i \nu t+i k \cdot r_{j}}+a d j .
$$

Similar to what we did in Eq. (6), here the ir Rabi frequency $\Omega_{j}=\bar{\Omega} \cos \theta_{j}$, where $\bar{\Omega}$ is given by Eq. (7).

Considering the case of an exact ir resonance on the $|d\rangle$ to $|c\rangle$ and $|c\rangle$ to $|b\rangle$ transitions (and that other vibrational transitions are sufficiently detuned that we can neglect pumping other vibrational states) and assuming all molecules are localized in a small volume, the eigenstates of Eq. (22) are given by

$$
\begin{gathered}
| \pm\rangle_{j}=\frac{1}{\sqrt{2}}\left[e^{i \phi}|c\rangle \pm \frac{1}{\sqrt{2}}\left(e^{2 i \phi}|b\rangle+|d\rangle\right)\right], \\
|0\rangle_{j}=\frac{1}{\sqrt{2}}\left(e^{2 i \phi}|b\rangle-|d\rangle\right),
\end{gathered}
$$

which are the usual dark or bright states of eigenvalues

$$
\begin{gathered}
V_{j}| \pm\rangle_{j}=\hbar \Omega_{j}| \pm\rangle_{j}, \\
V_{j}|0\rangle_{j}=0 .
\end{gathered}
$$

We prepare all molecules initially to be in the $|d\rangle$ state

$$
|d\rangle_{j}=\frac{1}{\sqrt{2}}\left[-|0\rangle_{j}+\frac{1}{\sqrt{2}}\left(|+\rangle_{j}-|-\rangle_{j}\right)\right]
$$

so that at a later time $t$ we have

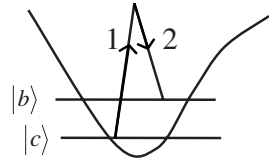

(a)

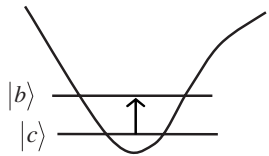

(b)
FIG. 10. (a) Preparation of $\rho_{b c}$ by ordinary indirect Raman; (b) $\rho_{b c}$ by direct ir-driven.

$$
\begin{aligned}
|\Psi(t)\rangle_{j}= & \frac{1}{\sqrt{2}}\left[-|0\rangle_{j}+\frac{1}{\sqrt{2}}\left(e^{-i \Omega_{j^{\dagger}}}|+\rangle_{j}-e^{-i \Omega_{j} t}|-\rangle_{j}\right)\right] \\
= & -e^{2 i \phi} \sin ^{2}\left(\frac{\Omega_{j}}{2} t\right)|b\rangle_{j}-\frac{e^{2} i \phi}{\sqrt{2}} \sin \left(\Omega_{j} t\right)|c\rangle_{j} \\
& +\cos ^{2}\left(\frac{\Omega_{j}}{2} t\right)|d\rangle_{j} .
\end{aligned}
$$

In the limit of small effective pulse area $(\Omega \tau \ll 1$, where $\tau$ is the ir pulse duration), the coherence $\rho_{b d}=-e^{2 i \phi}\left(\Omega_{j} \tau / 2\right)^{2}$; note that this quantity is quadratic in the effective pulse area, which is already assumed to be small. Substituting $\Omega_{j}$ $=\bar{\Omega} \cos \theta_{j}$, and summing over all molecules $j$, we obtain the average molecular orientation factor $\Sigma_{j} \cos ^{2} \theta_{j} / N$. Noting that Eq. (21) applies here in a straightforward way, if we simply replace $\rho_{b c}$ by $\rho_{b d}$ throughout, we conclude that for $N$ randomly oriented molecules $\Sigma_{j} \cos ^{2} \theta_{j}=N / 2$ the twophoton ir excitation is as efficient as Raman excitation when $\left(\Omega_{j} \tau\right)^{2} / 2 \approx \Omega_{1} \Omega_{2} \tau_{1,2} / \Delta$. However, Raman scattering off of the ir-generated coherence (between states $|d\rangle$ and $|b\rangle$ in Fig. 9, which represent a Raman overtone transition) will typically be less efficient than scattering off of the Ramangenerated coherence on a Raman fundamental transition (i.e., between states $|d\rangle$ and $|c\rangle$ ).

\section{Comparison of Raman coherence from indirect and direct processes}

In Eq. (28) the average molecular orientation factor $\cos ^{2} \theta_{j}$ may be replaced by $1 / 2$. We note that if we had attempted to prepare coherence via single ir photon absorption we would obtain $\cos \theta_{j}$ (instead of $\cos ^{2} \theta_{j}$ ) and the driving term in Eq. (28) would average to zero. This is the reason why two-photon absorption is required unless we orient the molecules.

Suppose we have an oriented ensemble, we could use single photon ir prepared coherence as in Fig. 10 just above; but what is the payoff? How big is $\rho_{b c}^{\mathrm{ir}} / \rho_{b c}^{\mathrm{Raman}}$ ? This ratio is roughly (electronic detuning)/(Rabi frequency), i.e., can be a very big number of the order of $10^{6}$ or more as in Fig. 10(b) below. Then $\cos \theta_{j}=1$, and the Raman coherence prepared by direct one-photon ir excitation of an oriented molecular ensemble $\rho_{b c} \approx \Omega \tau / 2$. This shows the payoff of molecular orientation: The resultant coherence can be substantially higher (linear in the effective pulse are, not quadratic) compared to the indirect (two-photon) excitation. In fact, comparing $\rho_{b c} \approx \Omega \tau / 2$ to Eq. (20) we find the ratio $\rho_{b c}^{\text {ir }} / \rho_{b c}^{\text {Raman }}$ $\cong\left|\Omega \tau \Delta / \Omega_{1} \Omega_{2} \tau_{1,2}\right|$. When all (one-photon) Rabi frequencies 


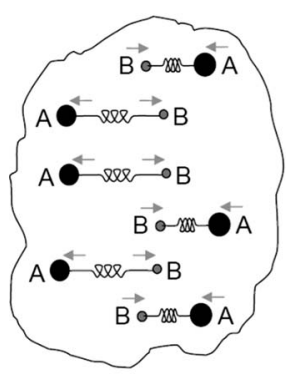

(a)

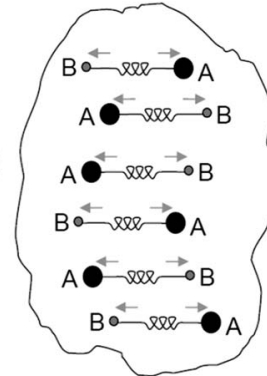

(b)

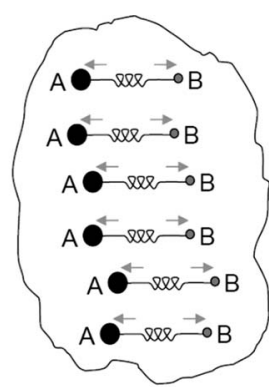

(c)
FIG. 11. Molecular oscillations in an ensemble of randomly oriented molecules produced by direct one-photon ir excitation (a), or by Raman excitation (b). Part (c) shows molecular oscillations in an ensemble of perfectly oriented molecules; such oscillations can result from either mode of excitation. The molecules are shown schematically as stretching or compressing, such that the lighter nucleus $\mathrm{B}$ moves in the direction shown by the arrows.

and pulse durations are equal, $\rho_{b c}^{\text {ir }} / \rho_{b c}^{\text {Raman }} \cong \Delta / \Omega$ can be a very big number in the order of $10^{6}$ or more (when far from saturation).

In summary, if a molecular ensemble with substantial orientation is available, molecular coherence can be prepared efficiently by a direct one-photon ir drive. This fact is well known in the community that applies sum-frequency spectroscopy to naturally oriented molecules in the vicinity of surfaces and interfaces [25]. Here we want to point out that there is important physical difference between two coherence systems $\left(\left|\rho_{b c}\right|>0\right)$ : one consisting of oriented ir-driven molecules, and another one consisting of misoriented Ramandriven molecules. In principle, both can generate coherent Raman signal, with efficiency proportional to the molecular coherence $\rho_{b c}$. Figure 11 illustrates the difference [parts (c) and (b)]. The molecules are shown schematically as stretching or compressing, such that the lighter nucleus B moves in the direction shown by the arrows. Part (a) shows the situation where with the absence of orientation ir radiation is absorbed but does not lead to any macroscopic Raman coherence.

\section{ACKNOWLEDGMENTS}

We thank Dick Miles, Steve Harris, and Paul Corkum for helpful discussions, and acknowledge support form the Office of Naval Research, National Science Foundation (Grants No. PHY 354897 and No. 722800), Texas Advanced Research Program (Grant No. 010366-0001-2007), the Army Research Office (Grant No. W911NF-07-1-0475), and the Robert A. Welch Foundation (Grants No. A-1261 and No. A-1547).

\section{APPENDIX: FRANCK-CONDON REMINDER}

We outline evaluation of the probability amplitudes $C \alpha(t)$ in the molecular wave function of Eq. (1) when subjected to fs laser pulses that induce transitions from the ground electronic state to excited states, denoted in Fig. 3 by Gd, Ex1, and Ex2, respectively.

$$
\Psi(X, \mathbf{r}, \mathbf{t})=\sum_{\alpha=g, 1,2} \mathbf{C}_{\alpha}(\mathbf{t}) \psi_{\alpha}(\mathbf{r}) \Phi_{\alpha}(\mathbf{X}, \mathbf{t}),
$$

where $\alpha$ designates the electronic state, $X$ the internuclear distance. For the $\mathrm{Gd} \rightarrow \mathrm{Ex} 1$ transition, the interaction potential $V(t)$ has the same form as Eq. (5), but with the ir laser field replaced by $E_{1}(t)$, the field of the laser inducing the transition; for the Ex $1 \rightarrow \mathrm{Ex} 2$ transition, and analogous form holds. The associated Schrödinger equation, with decay terms added, yields

$$
\begin{gathered}
\dot{C}_{g}=-\frac{i}{\hbar}\left\langle g\left|V_{e}(t)\right| 1\right\rangle C_{1}(t)-\gamma_{g} C_{g} \\
\dot{C}_{1}=-\frac{i}{\hbar}\left\langle 1\left|V_{e}(t)\right| g\right\rangle C_{g}(t)-\frac{i}{\hbar}\left\langle 1\left|V_{e}(t)\right| 2\right\rangle C_{2}(t)-\gamma_{1} C_{1}, \\
\dot{C}_{2}=-\frac{i}{\hbar}\left\langle 2\left|V_{e}(t)\right| 1\right\rangle-\gamma_{2} C_{2} .
\end{gathered}
$$

Here

$$
\langle g|V| 1\rangle=f_{g, 1}(t) \int d \mathbf{r} \psi_{\mathbf{g}}^{*}(\mathbf{r}) \mathbf{V}(\mathbf{t}) \psi_{\mathbf{1}}(\mathbf{r}) .
$$

In accord with the Franck-Condon approximation, the $X$ dependence of the electronic wave functions is neglected. The vibrational wave packet overlaps integral

$$
f_{g, 1}(t)=\int d X \Phi_{g}(X, t) \Phi_{1}(X, t) .
$$

A similar term pertains to the $\alpha=1,2$ pair of electronic states. We are chiefly concerned with the coherent superposition created by the ir pulse, given in Eq. (6): $\Phi_{g}(X, t)$ $=\Phi_{ \pm}(X, t)$. For our present heuristic purpose, in accord with Fig. 3, we make three simplifying assumptions:

(a) We assume that the fs pulses, $E_{1}(t)$, are essentially of "top-hat" form between $t_{0}$ and $t_{0}+T$, and that $\nu_{1}$ is resonant with a vibrational level in the Ex1 excited electronic state.

(b) We assume the Franck-Condon factor of Eq. (A4), $f_{g, 1}\left(t_{0}\right)$, is sizable for $\Phi_{g}=\Phi_{+}$but negligible for $\Phi_{g}=\Phi_{-}$; hence, for the associated Rabi frequencies, $\Omega_{+} \gg \Omega_{-}$.

(c) We neglect decay terms $\gamma_{g}$ and $\gamma_{1}$, which are much smaller that $1 / T$.

With these approximations, Eqs. (A2a)-(A2c) reduce to the form given in Eqs. (10a) and (10b) of the text, wherein + and - components correspond to the $A B$ and $B A$ orientations, respectively. 
[1] E. L. Eliel, Conformational Analysis (American Chemical Society, Washington, DC, 1981).

[2] Y. R. Shen, The Principles of Nonlinear Optics (Wiley, New York, 2002)

[3] For recent reviews, see H. Stapelfeldt and T. Seideman, Rev. Mod. Phys. 75, 543 (2003); D. Herschbach, Eur. Phys. J. D 38, 3 (2006).

[4] Note that anisotropy created by alignment behaves like a double-headed arrow whereas that created by orientation behaves like a single-headed arrow.

[5] P. R. Brooks and E. M. Jones, J. Chem. Phys. 45, 3449 (1966).

[6] R. J. Beuhler, R. B. Bernstein, and K. H. Kramer, J. Am. Chem. Soc. 88, 5331 (1966).

[7] C. H. Townes and A. L. Shawlow, Microwave Spectroscopy (McGraw-Hill, New York, 1955), pp. 248-250.

[8] H. J. Loesch, Annu. Rev. Phys. Chem. 46, 555 (1995).

[9] B. Friedrich and D. Herschbach, Comments At. Mol. Phys. 32, 47 (1995); Int. Rev. Phys. Chem. 15, 325 (1996).

[10] B. Friedrich and D. Herschbach, Phys. Rev. Lett. 74, 4623 (1995); J. Phys. Chem. 99, 15686 (1995).

[11] C. M. Dion, A. Keller, O. Atabek, and A. D. Bandrauk, Phys. Rev. A 59, 1382 (1999).

[12] J. Ortigoso, M. Rodriguez, M. Gupta, and B. Friedrich, J. Chem. Phys. 110, 3870 (1999).

[13] T. Seideman, Phys. Rev. Lett. 83, 4971 (1999); J. Chem. Phys.
115, 5965 (2001).

[14] B. Friedrich and D. Herschbach, J. Phys. Chem. A 103, 10280 (1999).

[15] S. Guerin, L. P. Yatsenko, H. R. Jauslin, O. Faucher, and B. Lavorel, Phys. Rev. Lett. 88, 233601 (2002).

[16] S. E. Harris, Phys. Today 50 (9), 36 (1997).

[17] M. O. Scully and M. S. Zubairy, Quantum Optics (Cambridge University Press, Cambridge, England, 1997).

[18] V. A. Sautenkov, C. Y. Ye, Y. V. Rostovtsev, G. R. Welch, and M. O. Scully, Phys. Rev. A 70, 033406 (2004).

[19] G. Herzberg, Molecular Spectra and Molecular Structure: I. Spectra of Diatomic Molecules (Van Nostrand-Reinhold, New York, 1950).

[20] K. Kirby and D. L. Cooper, J. Chem. Phys. 90, 4895 (1989).

[21] G. I. Petrov, R. Arora, V. V. Yakovlev, X. Wang, A. V. Sokolov, and M. O. Scully, Proc. Natl. Acad. Sci. U.S.A. 104, 7776 (2007).

[22] D. Pestov, G. O. Ariunbold, X. Wang, R. K. Murawski, V. A. Sautenkov, A. V. Sokolov, and M. O. Scully, Opt. Lett. 32, 1725 (2007).

[23] Fam Le Kien, J. Q. Liang, M. Katsuragawa, K. Ohtsuki, K. Hakuta, and A. V. Sokolov, Phys. Rev. A 60, 1562 (1999).

[24] A. V. Sokolov and S. E. Harris, J. Opt. B Quantum Semiclassical Opt. 5, R1 (2003).

[25] Y. R. Shen, Nature (London) 337, 519 (1989). 